\title{
RNA Sequencing of Neuropathic Pain in the Anterior Cingulate Cortex after Nerve Injury
}

yu zhang ( $D$ 1162386063@qq.com)

Xiangya Hospital Central South University https://orcid.org/0000-0002-7687-4420

Shiwei Jiang

Central South University Xiangya Medical College: Central South University Xiangya School of Medicine

Fei Liao

People's Hospital of Yuxi City

Zhifeng Huang

Xiangya Hospital Central South University

Xin Yang

Xiangya Hospital Central South University

Yu Zou

Xiangya Hospital Central South University

Xin $\mathrm{He}$

Xiangya Hospital Central South University

Qulian Guo

Xiangya Hospital Central South University

Changsheng Huang

Xiangya Hospital Central South University https://orcid.org/0000-0003-0535-1865

\section{Research Article}

Keywords: neuropathic pain, CCl, RNA sequence, Anterior Cingulate Cortex, chemokines.

Posted Date: September 9th, 2021

DOI: https://doi.org/10.21203/rs.3.rs-861152/v1

License: (c) (i) This work is licensed under a Creative Commons Attribution 4.0 International License.

Read Full License 
1 RNA Sequencing of Neuropathic Pain in the Anterior

4 Yu Zhang ${ }^{1}$, Shiwei Jiang ${ }^{2}$, Fei Liao ${ }^{3}$, Zhifeng Huang ${ }^{1}$, Xin Yang $^{1}$, Yu Zou ${ }^{1}$, Xin

$5 \quad \mathrm{He}^{1}$, Qulian Guo ${ }^{1,4}$, Changsheng Huang* 1,4

6 Author affiliations:

7 'Department of Anesthesiology, Xiangya Hospital, Central South University,

8 Changsha, China

$9 \quad{ }^{2}$ Medical College of Xiangya, Central South University, Changsha, China.

10 'Department of Anesthesiology, People's Hospital of Yuxi City, Yuxi, China.

$11{ }^{4}$ National Clinical Research Center for Geriatric Disorders, Xiangya Hospital,

12 Central South University, Changsha, China

${ }^{*}$ Corresponding author: Changsheng Huang

15 Corresponding address: Department of Anesthesiology, Xiangya Hospital,

16 Central South University, 87 Xiangya Road, Changsha 410008, Hunan,

$17 \quad$ China

Tel/Fax: +86 73184327413

19 E-mail address: changsheng.huang@csu.edu.cn 


\section{Abstract}

2 Background: Neuropathic pain is a troublesome pathological condition

3 without suitable treatments. Anterior Cingulate Cortex (ACC) is a core brain

4 region to process pain emotion. In this study, we performed RNA sequencing

5 analysis to reveal transcriptomic profiles of the ACC in a rat chronic

6 constriction injury $(\mathrm{CCl})$ model.

7 Results: A total of 1628 differentially expressed genes (DEGs) were identified

8 by comparing the sham-operated rats and rats of 12 hours, $1,3,7$ and 14 days

9 after surgery, respectively. Most of the DEGs were involved in inflammatory

10 and immune process. Although these inflammatory-related DEGs were

11 generally increased after $\mathrm{CCl}$, they demonstrated different kinetics in

12 time-series expression with the development of neuropathic pain affection.

13 Specifically, the expression of $\mathrm{Ccl} 5, \mathrm{Cxc} / 9$ and $\mathrm{Cxcl} 13$ were kept going up after

$14 \mathrm{CCl}$, indicating a potentially effect of these genes on initiation and

15 maintenance of neuropathic pain affection. The expression of $\mathrm{Ccl} 2, \mathrm{Cc} / 3, \mathrm{Ccl}$,

$16 \mathrm{Cc} / 6$ and $\mathrm{Ccl} 7$ were initially upregulated at 12 hours after $\mathrm{CCl}$ and then they fell

17 back after that. Similarly, the expression of Rac2, Cd68, Icam-1, Ptprc, Itgb2,

18 Fcgr2b were rised at 12 hours and 1 day, but fell back at 3 days after $\mathrm{CCl}$.

19 However, the expression of all of the above two clusters of genes were

20 increased again at 7 days after $\mathrm{CCl}$, when the neuropathic pain affection was

21 developed. The initial increase of these genes may indicate an early response of ACC to nerve injury, whereas the later increase of these genes may indicate 
1 their involvement in the developing of neuropathic pain affection. Gene

2 Ontology analysis, KEGG pathway enrichment and interaction network

3 analysis further showed a high connectivity degree among these chemokine

4 targeting genes. Similar expressional changes of these genes were also found

$5 \quad$ in the rat spinal dorsal taking charge of the processing of nociception.

6 Conclusions: Our results indicate chemokines and their targeting genes in

7 ACC may be differentially involved in the initiation and maintenance of

8 neuropathic pain affection. These genes could be the target not only the

9 nociception but also the pain affection subsequent to nerve injury.

Keywords: neuropathic pain; CCI; RNA sequence; Anterior Cingulate Cortex;

11 chemokines.

\section{Introduction}

Neuropathic pain caused by the injury or disease of somatosensory nervous system is a pathological condition, which brings great trouble to patients. According to the data of the International Association for the study of pain in 2019 , about $10 \%$ of the world's population suffered from neuropathic pain ${ }^{1}$. It is of great significance to deeply study the pathogenesis of neuropathic pain and explore effective treatment drugs for the development of human health.

ACC located in the forebrain is an important part of limbic system. Accumulating evidence shows that $A C C$ is a core brain region to process pain emotion. Clinical studies have shown that the structure and function of ACC in 
1 patients with chronic pain change significantly ${ }^{2-4}$.The synaptic plasticity is the

2 key mechanism of the occurrence and development of chronic pain in ACC

3 supported by a large number of animal experiments ${ }^{5,6}$. Peripheral inflammatory

4 pain or neuropathic pain can cause significant changes in synaptic

5 transmission and morphological plasticity of ACC neurons ${ }^{7-9}$, and blocking this

6 abnormal plasticity of ACC plays a significant analgesic effect ${ }^{10,11}$. Long-term

7 potentiation (LTP) is a form of synaptic plasticity that have been studied in the

8 context of learning, memory, and chronic pain ${ }^{5,12,13}$. LTP includes two forms,

9 one is presynaptic form of LTP (pre-LTP) and the other is postsynaptic form of

10 LTP (post-LTP) ${ }^{14}$. In ACC, post-LTP works in a glutamatergic

11 NMDA-dependent manner to sustain the pain affection, which involves

12 adenylyl cyclase type 1 (AC1) and a-amino-3-hydroxy-5-methyl-4-isoxazole

13 propionic acid (AMPA) receptor ${ }^{10}$. However, pre-LTP is triggered by kainate receptors, rather than NMDA receptors, involved in anxiety-like behaviors ${ }^{15}$. reveal the mechanism underlying psychiatric disorders ${ }^{16-18}$, diurnal rhythms ${ }^{19}$ and cognitive dysfunction ${ }^{20}$, however, with few in neuropathic pain. A recent study revealed a whole transcriptome in the spinal cord, ACC, and amygdala

19 following spinal nerve ligation (SNI). But the comparison was just between sham-surgery and SNI-7days mice ${ }^{21}$. In order to explore a comprehensive

21 understanding of the ACC cells under neuropathic pain conditions, our group

performed RNA sequencing analysis to distinguish DEGs from the 
1 sham-operated rats and rats of 12 hours, $1,3,7$ and 14 days after $\mathrm{CCl}$

2 respectively. We found the DEGs demonstrated different kinetics in time-series

3 expression with the development of neuropathic pain affection. Through Gene

4 Ontology (GO) analysis, KEGG pathway enrichment and protein-protein

5 interaction (PPI) network analysis, our findings supported chemokines and

6 their targeting genes in ACC may be differentially involved in the initiation and

7 maintenance of neuropathic pain affection. These genes could be the target

8 not only the nociception but also the pain affection subsequent to nerve injury.

\section{RESULT}

2.1.Behavioral Characterization of The Rats After CCI

We performed $\mathrm{CCl}$ in rats and then measured PWMT and PWTL of the rats from 12 hours to 14 days. Compared with the sham rats, the PWMT and PWTL of the $\mathrm{CCl}$ rats decreased from 3 days to 14 days (Fig.1A-B). SPT showed a decrease intake of sucrose in $\mathrm{CCl}$ rats (Fig $1 \mathrm{C}) . \mathrm{CCl}$ rats spent more time in the light area to escape the stimulation in the PEAP test, while sham rats were willing to stay in the dark area (Fig 1D). These results showed that the neuropathic pain model was successfully established.

\subsection{Transcripts Regulated in the ACC of Rat After CCI}

We performed RNA sequencing analysis to reveal transcriptomic profiles of the $\mathrm{ACC}$ in $\mathrm{CCl}$ rats. The transcriptome data were generated from the rats of 12 
1 hours, $1,3,7$ and 14 days after $\mathrm{CCl}$ and sham-operated rats. Figure 2B

2 showed the quality control of sequencing data of each sample. On average,

3 about 60 million clean reads were collected and a mapping rate of round

4 93 97\%. The Q20 ratio were all above 94\%. Then we calculated the

5 correlation value between every two samples based on normalized expression

6 results and draw a correlation heat map (Fig. 2A).

7 Our data revealed that there were 1628 DEGs with the cut-offs of fold

8 change $>1.5$ and $P$-value $<0.05$. The representative distributions of genes up-

9 or down-regulated were shown in the volcano as Fig. 3A-E. As the Venn

10 diagram showed (Fig. 3F), 38 DEGs were shared in the five time points. On 12

11 hours after $\mathrm{CCl}$ there were 349 genes differentially expressed with 150

12 up-regulated and 199 down-regulated. On day 1, 3,7 and 14 , the ratio of

13 up-regulated DEGs / all DEGs in the same day were 234/428, 201/524,

$14403 / 672$ and 562/748 respectively. And the ratio of down-regulated DEGs were

15 194/428, 333/524, 269/672 and 196/748 (Fig. 3G). In general, within 14 days,

16 the number of up-regulated genes in the $\mathrm{ACC}$ after $\mathrm{CCl}$ basically showed an

17 upward trend, reaching a maximum at 14 days after nerve injury, and only

18 slightly decreased on the third day after nerve injury; while the number of

19 down-regulated genes was basically unchanged within 1 days after the nerve

20 injury, and the number increased significantly on the $3^{\text {rd }}$ day after the injury,

21 and then gradually decreased. These findings revealed that there were a large

22 number of unique DEGs expression at different stages of pain. 
2 2.3.Gene Ontology Analysis of DEGs

3 To further understand the specific functions of DEGs in ACC of rats after $\mathrm{CCl}$,

4 we performed GO Analysis to analyze the biological process, cellular

5 component and molecular function of the DEGs. GO Analysis identified the

6 biological processes enriched with "immune system process", "defense

7 response", "regulation of immune system process", "cell adhesion" and

8 "cytokine production", suggesting that strong immune and inflammatory

9 response occurred in $\mathrm{ACC}$ after $\mathrm{CCl}$. The cellular components were mainly

10 "cell periphery", "vesicle”, "intrinsic component of plasma membrane", "integral

11 component of plasma membrane" and "plasma membrane protein complex"

12 indicating that multiple membrane components involved in neuropathic pain.

13 The molecular function terms focused on "protein binding", "signaling receptor

14 binding", "G protein-coupled receptor binding", "antigen binding" and "CCR

15 chemokine receptor binding". The GO analysis revealed that the immune and

16 inflammatory responses were key physiological process on the occurrence

17 and development of neuropathic pain (Fig 4A-C).

\subsection{Analysis of KEGG Pathways}

Then we analyze the DEGs through KEGG pathway enrichment to identify the

21 major signaling pathways involved in the neuropathic pain (Fig 5). Among the

22 top30 pathways, the DEGs were enriched in "TNF signaling pathway", 
1 "Phagosome", "NF-kappa B signaling pathway", "Cytokine-cytokine receptor

2 interaction", "Complement and coagulation cascades", "Chemokine signaling

3 pathway", "Cell adhesion molecules (CAMs)" and "Antigen processing and

4 presentation", which similarly indicated that the cytokines and complements

5 participated in neuropathic pain largely. Besides, some pathways concerning

6 infection or immune system diseases were also enriched, for instance, "Viral

7 myocarditis" and "Type I diabetes mellitus". These results showed that

8 immune and inflammatory responses were pivotal in $\mathrm{ACC}$ after $\mathrm{CCl}$.

\subsection{Different Kinetics in Time-series Expression of DEGs in ACC after $\mathrm{CCl}$}

11 Except for some shared DEGs, a large number of unique DEGs existed at different stages of pain. We classified all DEGs into 50 clusters according to the time series. We found there were 6 clusters of gene expression profiles showing statistical difference, namely profiles 7, 12, 27, 36, 39 and 47. Except for profile 12, the other 5 profiles showed an overall upward trend. Surprisingly, we found the profile 27 and 36 were up-regulated successively. The profile 36 DEGs were up-regulated to a small peak on 12 hours, fell back in day 1 , while the DEGs of profile 27 started to be up-regulated at 12 hours, reached a small 
1 cellular component against them. As Supplementary Fig.1A showed, the

2 enriched biological processes of profile 36 were those representing immune

3 processes, they were "immune response", "response to cytokine", "response to

4 interferon-gamma" and "cellular response to interferon-gamma". The role of

5 chemokines in the enriched molecular function terms were particularly

6 prominent, including "cytokine receptor binding", "cytokine activity",

7 "chemokine receptor binding", "chemokine activity" and "CCR chemokine

8 receptor binding". The enriched cellular components were mainly in the

9 nucleus, such as "nuclear chromosome", "chromosome" and "chromatin". In

10 the profile 27 , the enriched biological processes were those representing

11 immune processes, they were "immune system process", "cell activation",

12 "lymphocyte aggregation", "leukocyte aggregation", "mononuclear cell

13 proliferation", “lymphocyte proliferation", "leukocyte proliferation" and "T cell

14 proliferation". The enriched molecular function terms were "protein binding",

15 "enzyme binding", "kinase binding" and "MHC class II protein complex binding".

16 The enriched cellular components were extranuclear, they were "membrane",

17 "cell periphery" and "plasma membrane". As for the DEGs in the profile 39 , the

18 biological processes were "immune system process", "immune response",

19 "positive regulation of immune system process" and "cell activation", the

20 molecular function terms were "anion binding", "carbohydrate derivative

21 binding" and "ribonucleoside binding", and the cellular components were

22 "protein-containing complex" "plasma membrane protein complex", 
"chromosome" and "nuclear chromosome".

To identify the major signaling pathways involved in the formation and development of neuropathic pain, we continued to analyze DEGs in profile 27 、 36 and 39 via KEGG pathway enrichment. Our results showed that the significantly enriched DEGs were in the classifications of "Cytokine-cytokine receptor interaction", "chemokine signaling pathway", "cell adhesion molecules (CAMs)" and other cell-defense related pathways (Supplementary Fig.1B). Among them, cytokine—related pathways were outstanding in the profile 36 and 39, while immune cell-related pathways were predominant in the profile 27 , such as "Fc gamma R-mediated phagocytosis", "B cell signaling pathway", "Leukocyte transendothelial migration" and "T cell receptor signaling pathway". These results indicated that the initial increase of these DEGs may indicate an early response of $A C C$ to nerve injury, whereas the later increase of these genes may indicate their involvement in the developing of neuropathic pain affection.

\subsection{PPI Network Analysis of DEGs}

Then we performed function analysis of the DEGs involving in the above pathways from the 3 profiles by literature retrieval and found that more than half were chemokine family (Table 1). CC motif chemokine ligand 5 (Cc/5) 、 C-X-C Motif Chemokine Ligand $9(\mathrm{Cxc} / 9)$ and $\mathrm{Cxc} / 13$ were in profile 39, while Cc/2, Cc/3, Cc/4, Ccl6 and Ccl7 were in profile 36. In profile 27, there were 
1 some inflammatory and immune-related genes, for example Rac2, Cd68,

2 Icam-1, Ptprc, Itgb2. Besides, many of them had been known functions in

3 neuroinflammation or nerve injury. Since genes in profile 27 and 36 changed

4 successively, we conducted PPI network analysis to investigate the underlying

5 relationship between them. The PPI network demonstrated a complex

6 interaction among genes containing 62 nodes and 282 edges with enrichment

7 p-value<1.0e-16. Following genes showed high connectivity degrees:

$8 \quad$ Ptprc(degree=35), Cd68(degree=26), Cc/2(degree=19), Vav1(degree=17),

9 Rac2(degree=17), Itgb2(degree=17), Fcgr2b(degree=17), Icam-1(degree=15),

$10 C c / 3($ degree=12), $C c / 4($ degree=11), $\quad C c / 7($ degree=10), etc.. (Fig 7). These

11 results suggested that chemokine targeting genes in ACC may be differentially

12 involved in the initiation and maintenance of neuropathic pain affection.

14 2.7. Similar expressional changes of chemokines in ACC and spinal cord

15 In order to know the specific expression patterns of each chemokine in ACC,

16 we made a bar chart to show the expression levels of them at different stages.

17 As figure 8B showed, all of the chemokines changed variously. Chemokines

18 have been proved involving in spinal cord in charge of the processing of

19 nociception after nerve injury ${ }^{31}$, thus we compared the gene expression

20 patterns of chemokines in ACC with that in spinal cord. The quality control of

21 sequencing data of each spinal cord sample was shown in supplementary

22 table. As the heatmap showed (Fig 8A), all the chemokines were in general 
1 upward trends and appeared similar expressional changes. It suggested that

2 the DEGs may be differentially involved in the initiation and maintenance of

3 neuropathic pain affection. These genes could be the target not only the

4 nociception but also the pain affection subsequent to nerve injury.

\section{Discussion}

7 Pain includes pain affection and emotional perception. ACC is a key brain

8 region to regulate pain. In this study, we performed RNA-seq to reveal dynamic

9 transcriptomic profiles of the ACC from $\mathrm{CCl}$ rats. We found $1628 \mathrm{DEGs}$ were

10 mainly involved in inflammatory and immune process. Although these

11 inflammatory-related DEGs were generally increased after $\mathrm{CCl}$, they

12 demonstrated different kinetics in time-series expression with the development

13 of neuropathic pain affection. GO analysis, KEGG analysis and PPI network

14 analysis showed a high connectivity degree among these chemokine targeting

15 genes. Similar expressional changes of these genes were also found in the rat

16 spinal dorsal which takes charge of the processing of nociception. Our results

17 indicate that chemokine targeting genes in ACC may be differentially involved

18 in the initiation and maintenance of neuropathic pain affection. These genes

19 could be the target not only the nociception but also the pain affection

20 subsequent to nerve injury.

21 ACC activation is closely related to inflammatory response. Harrison

22 found ACC activation was associated with increased IL-6 accompanied with 
1 fatigue and confusion after healthy individuals receiving typhoid vaccination ${ }^{32}$.

2 ACC activation also existed in stress-induced inflammatory responses ${ }^{33}$.

3 Besides, microglial activation was detected by labeling translocator protein

4 (TSPO) with positron emission tomography (PET) in major depressive disorder

5 patients, and elevated TSPO levels were observed in their $\mathrm{ACC}^{33,34}$. It is clear

6 that neuropathic pain is associated with a profound neuroinflammation and

7 immune response, in which chemokines work to some extent. Accumulating

8 evidence shows that chemokines work in the DRG and spinal cord under

9 chronic pain conditions ${ }^{31}$. However, there are few studies on chemokines in

10 brain. Our results showed that some chemokines including $\mathrm{Cc} / 5, \mathrm{Cxc} / 9$ and

11 Cxc/13 were up-regulated persistently in ACC during the development and

12 maintenance of neuropathic pain. Recent studies revealed increased Cxcl13

13 and its receptor C-X-C chemokine receptor type 1 ( Cxcr5) triggered

14 neuropathic pain-related conditioned place aversion. Cxcl13 mRNA was found

15 gradually increasing in ACC from 1 to 10 days after surgery, which

16 corresponded to our results. Cxcll13/Cxcr5 are also upregulated in the

17 trigeminal ganglion (TG), DRG and spinal cord on pain conditions. Besides,

18 the pain hypersensitivity can be attenuated through inhibition of $\mathrm{Cxcl} 13 / \mathrm{Cxcr} 5$

19 signaling ${ }^{31}$. Ccl5, predominantly recruiting and activating $\mathrm{T}$ lymphocytes and

20 NK cells, was found to be localized in infiltrating lymphocytes of the

21 blood-brain barrier (BBB) while Cxcl9 localized in the cerebral microvessels

22 and glial cells under pathological conditions ${ }^{35}$.It has been confirmed that Ccl5 
1 is closely related to pain in DRG, spinal cord ${ }^{36}$ and the injured nerve ${ }^{37}$.

2 Intraperitoneal injection of $\mathrm{Ccl} 5$ receptor antagonist or knocking out $\mathrm{Ccl} 5$ gene

3 in rats can reduce the infiltration of macrophages and the release of

4 pro-inflammatory cytokines such as TNF $\alpha, \mathrm{IL}-1 \beta, \mathrm{IL}-6$ and IFN- $\gamma$ after nerve

5 injury ${ }^{37,38}$. Cxcl9 was up-regulated in spinal astrocytes after SNL, while

6 intrathecal injection of Cxcl9 or inhibition of spinal Cxcl9 was invalid for pain

7 relief ${ }^{39}$. It is of great significance to further study Ccl5 and Cxcl9 in ACC to

$8 \quad$ clarify their roles in neuropathic pain.

Previous reports suggested that chemokines may participate in recruiting immune cells dependent on time and context. For example, it was Ccl2/Ccr2

11 rather than $\mathrm{Ccl} 5 / \mathrm{Ccr} 5$ recruited monocytes to exacerbate inflammation in osteoarthritis $^{40}$. In atherosclerosis, Ccr2- monocytes rely on Ccr5, while Ccr2 ${ }^{+}$ monocytes dependent on Cx3cr1 to enter plaques ${ }^{41}$. Here ,we found C-C chemokine receptor type 1 ( $\mathrm{Ccr} 1$ ) ligands $(\mathrm{Ccl}$, $\mathrm{Cc} / 3, \mathrm{Ccl} 4, \mathrm{Ccl} 6, \mathrm{Ccl}$, except

15 for $\mathrm{Ccl} 5$ ) were up-regulated in ACC twice, before 12 hours and after 7 days respectively. Studies reported that the levels of $\mathrm{Ccl} 2, \mathrm{Ccl} 3, \mathrm{Ccl} 4, \mathrm{Ccl} 6$ and $\mathrm{Ccl} 7$

17 significantly increased in spinal cord after $\mathrm{CCl}$. Also, Ccl2, Ccl6 and Ccl7 were up-regulated in DRG in $\mathrm{CCl}$ rats. Single and multiple intrathecal injection of

$19 \mathrm{~J} 113863$ (Ccr1 antagonist) can relief mechanical and thermal pain. Moreover, repeated intrathecal injections of J11386 can reduce the activation and 21 infiltration of microglia, monocytes, neutrophils, lymphocytes, etc., thereby 
1 regulating the levels of pronociceptive ( $\mathrm{IL}-1 \mathrm{~b}, \mathrm{IL}-6$ and $\mathrm{IL}-18$ ) and

2 antinociceptive properties (IL-1RA) $)^{42}$.

3 Another profile of DEGs also appeared to be up-regulated twice, but the

4 first time was from 12 hours to 1 day after $\mathrm{CCl}$. Compared with DEGs in above

5 profile, the GO analysis and KEGG pathway enrichment of this profile showed

6 that 1 ) the biological processes about various immune cells activation,

7 proliferation, aggregation and adhesion were particularly prominent. 2) cellular

8 component were "membrane" " cell surface" v"external side of plasma

9 membrane" and "cell periphery" rather than mainly in the nucleus. 3)molecular

10 function were "protein binding" 、 "enzyme binding" 、"MHC class II protein

11 binding" and "MHC class II protein complex binding" . 4) the signaling pathway

12 including "T cell receptor signaling pathway" 、 "Natural killer cell mediated

13 cytotoxicity"、"Leukocyte transendothelial migration"、"Fc gamma R-mediated

14 phagocytosis" and "B cell receptor signaling pathway" were mainly

15 immune-related. The results suggested the expression of these DEGs in

16 profile 27 from $\mathrm{ACC}$ after $\mathrm{CCl}$ were inseparable from the role of chemokines. In

17 profile 27, the DEGs included Rac2, Cd68, Icam-1, Ptprc, Itgb2, etc.. Rac2 is a

18 member of Rho Small GTPase family that can regulate the cytoskeletal

19 dynamics, cell shape, migration, adhesion, gene transcription and signal

20 transduction $^{43}$. A previous study identified Rac2 was a crucial gene

21 participating in the pathological process of neuropathic pain ${ }^{44,45}$, which might

22 be related to its regulation of inflammation and immune response ${ }^{46,47}$. Cd68 is 
1 a lysosome marker associated with phagocytosis expressed in actived

2 microglia ${ }^{48}$. In the early stages of systemic inflammation, microglia were

3 attracted to vessels to protect the BBB integrity by Ccl5-Ccr5 signaling. Once

4 the BBB was damaged, microglia transformed to a reactive phenotype

5 expressing Cd68 that amplified the neuroinflammation ${ }^{49}$. Icam-1 was identified

6 a cell surface glycoprotein to regulate inflammation and injury resolution ${ }^{50}$.

7 After SNI, Icam-1 was up-regulated in small extracellular vesicles ${ }^{51}$. Besides,

8 Icam-1 expressed on vascular endothelium could recruit opioid-containing

9 immune cells to promote analgesia ${ }^{52}$. And also, Icam-1 was found closely

10 related to chemokines. After Ccl7 treatment, Icam-1 was significantly

11 increased in human umbilical endothelial cells (HUVECs) ${ }^{53}$. On the other hand,

12 Icam-1 could inhibit the expression of $\mathrm{Ccl} 2$ by up-regulation of miR-124, and

13 thereby promote macrophage polarization ${ }^{54}$. Ptprc, namely Recombinant

14 Protein Tyrosine Phosphatase Receptor Type C, acts as a positive regulator of

15 T-cell coactivation. Ptprc was found up regulation in microglia isolated from

16 LPS-injected mice ${ }^{55}$. Itgb2(Cd18) was a transmembrane glycoprotein enable

17 to aggravate the injury after $\mathrm{SCl}^{56}$. Accordingly, the initial increase of these

18 DEGs may indicate an early response of ACC to nerve injury, whereas the later

19 increase of these genes may indicate their involvement in the developing of

20 neuropathic pain affection. These results indicated that chemokines and their

21 targeting genes in ACC may be differentially involved in the initiation and

22 maintenance of neuropathic pain affection. These genes could be the target 
not only the nociception but also the pain affection subsequent to nerve injury.

As our results showed the expression pattern of chemokines in ACC was similar to spinal cord after $\mathrm{CCl}$. The connection between ACC and spinal cord was complicated and still unclear. Nociceptive information was transmitted to spinal cord horn via primary afferent sensory neurons. The nociceptive neurons in spinal cord conveyed the ascending signals to supraspinal areas, such as thalamus and ACC. In ascending pain regulation system, ACC doesn't receive the projections from spinal cord. However, the nerve projections exist in ACC-spinal cord pathway. Chen injected the retrograde tracer Fluoro-Gold into the cervical spinal cord of mouse, and found the labeled neurons mostly were in layer $\mathrm{V}$ of $\mathrm{ACC}$. After injecting an anterograde neuronal tracer, namely phaseolus vulgaris leucoagglutinin (Pha-L) and gene-edited rabies virus respectively into ACC, the labeled neurons were mainly in the lamina I-III of the spinal cord. Moreover, expression of GluA1 and potentiated AMPA receptor (AMPAR)-mediated postsynaptic responses were increasing in the ACC—spinal cord projecting neurons after nerve injury. Further research found that $\mathrm{Ca}_{2}{ }^{+}$permeable AMPAR antagonist NASPM could reverse the potentiation of postsynaptic responses and pain sensitization ${ }^{57-59}$.In addition, ACC-brainstem-spinal cord pathway is another descending pain modulating pathway, involving periaque ductal gray (PAG) and rostromedial ventral medulla $(\mathrm{RVM})^{60}$. It still needs great efforts to make clear the connection between ACC and spinal cord. Elucidating the possible mechanism about 
1 chemokines between ACC and spinal cord may provide us with profound

2 insights into the mechanisms of neuropathic pain.

As we all know, one of the main reasons for the high prevalence of neuropathic pain is its unclear pathogenesis and lack of effective treatments. Unlike opioids and nonsteroidal anti-inflammatory drugs (NSAIDs), which can effectively relieve nociceptive pain, the drugs currently used for the treatment of neuropathic pain, including tricyclic antidepressants, 5-hydroxytryptamine / norepinephrine reuptake inhibitors and ion channel modulators / blockers, are often effective only in some patients or in a period of the course of the disease $^{61}$. It is of great significance to deeply study the pathogenesis of neuropathic pain and explore effective treatment drugs. Our results showed chemokines and their targeting genes were considerable treating targets for neuropathic pain. Maraviroc, Ccr5 antagonist, is the first chemokine receptor targeted drug approved by the FDA and is currently clinically used to treat HIV-1 infections. Maraviroc also have effects on reducing tumor growth ${ }^{62}$ and neuropathic pain ${ }^{63}$. Plerixafor, known as AMD3100, is in clinical use for hematopoietic stem cell mobilization ${ }^{64}$ and also can alleviate neuropathic pain $^{65}$.Except for drugs, neutralizing antibody can be used for treatments too. Mogamulizumab-kpkc, against Ccr4, has been approved by FDA to treat adults with Mycosis Fungoides and Sézary Syndrome ${ }^{66}$. Maybe it can be used in pain in the future. 


\section{4. Conclusion}

2 In conclusion, we found that ACC was not only involved in emotion

3 regulation, but also in connection with inflammatory and immune responses in

4 the occurrence and development of neuropathic pain. Moreover, chemokines

5 and their targeting genes in ACC may be differentially involved in the initiation

6 and maintenance of neuropathic pain affection. These genes could be the

7 target not only the nociception but also the pain affection subsequent to nerve 8 injury.

\section{Methods}

\subsection{Animals}

Male Sprague-Dawley (SD) rats weighing $220 \mathrm{~g}-250 \mathrm{~g}$ were purchased form Hunan SLAC Laboratory Animal Co., LTD., Changsha, China. Rats were raised in group of 3 per cage with a free access to food and water under a $12 \mathrm{~h}$ light/dark cycle in a suitable environment for temperature and humidity. All procedures were in accordance with the National Institutes of Health guide for the care and use of Laboratory animals and approved by Institutional Ethics Committee of Central South University.

\subsection{Model of $\mathrm{CCl}$}

$\mathrm{CCl}$ was performed in rats according to the method of Bennett and $\mathrm{Xie}^{22}$. After the rats were anesthetized with isoflurane, the left sciatic nerve was exposed 
1 and tied around by four snug ligatures (4-0) with the same tightness and

2 intervals. After ligation, the nerve was repositioned. In sham surgery rats, the

3 left sciatic nerve was just exposed without ligation.

4

\subsection{Behavioral assessment}

The paw withdrawal mechanical threshold (PWMT) and the paw withdrawal thermal latency (PWTL) of the rats were measured on 1 day before $\mathrm{CCl}$ and from 12 hours to 14 days after $\mathrm{CCl}$. The measured value before $\mathrm{CCl}$ was used as the basic threshold (BL). All the assessment were carried out after the rats were acclimated in specific individual chambers at least $30 \mathrm{~min}$. The PWMT was measured with Von Frey filaments (North Coast Medical, San Jose, CA, USA) ranging from $0.4 \mathrm{~g}-15 \mathrm{~g}$, as described in our previous study ${ }^{23,24}$. Briefly, the stimuli were applied vertically to the plantar surface of the left hind paw, and the minimal force that could cause three consistent withdrawal responses (lifting or licking) was considered as the PWMT. A thermal pain test instrument (Tes7370, Ugo Basile, Comerio, Italy) $)^{23,24}$ was used for the test of PTWL. In a brief, after the rats were habituated in the cage for $30 \mathrm{~min}$, a continuous heat stimuli was applied to their plantar surface of hindpaw to evoke withdrawal responses and meanwhile the timer recorded the latency. The cut-off time was set at 30 s and each rat was tested for three times with a 5 min interval. The three latencies were averaged as PTWL. 
1 Sucrose preference test (SPT) was performed according to a previous study ${ }^{25}$.

2 Rats were given $1 \%$ sucrose solution for $3-5$ days. Then, the experiment was

3 carried out on 1 day before $\mathrm{CCl}(\mathrm{BL}), 3,7$ and 14 days after $\mathrm{CCl}$. During the

4 test, rats were fed in single cage and given two bottles of water ( $1 \%$ sucrose

5 solution and water). The positions of sucrose solution and water were

6 exchanged every 12 hours. After 24 hours, the consumption of sucrose

7 solution and water was measured and the percentage of sucrose preference

8 was calculated.

9 Place escape/avoidance paradigm (PEAP) was performed on rats as 10 described previously ${ }^{26}$. The rats were placed in a $30 \times 30 \times 30 \mathrm{~cm}$ chamber and

11 moved freely for 30 minutes before test. The chamber was on top of a mesh

12 floor. One half of the chamber was painted black (dark area), and the other

13 was light area. A $60 \mathrm{~g}$ Von Frey filament was applied to the plantar surface of

14 the hindpaw every $15 \mathrm{~s}$ for $30 \mathrm{~min}$. The injured hindpaw was stimulated within

15 the dark area, and the hindpaw contralateral to $\mathrm{CCl}$ was stimulated within the

16 light area. The time spent in the light area throughout the test was recorded

17 and converted to a percentage to reflect the level of pain affect. All the

18 behavioral tests were accomplished by the investigators blind to the 19 experimental groups.

21 5.4. Total RNA Extraction and Purification 
1 Rats were sacrificed under deep anesthetization at different time points. The

2 ACC and the spinal cord were collected from each rat after perfusion with

3 phosphate-buffered saline (PBS) and immediately frozen by dry ice and then

4 stored at $-80^{\circ} \mathrm{C}$. Total RNA was extracted using RNeasy Micro Kit (Cat\# 74004,

5 Qiagen) following the manufacturer's instructions and checked for a RIN

6 number to inspect RNA integrity by an Agilent Bioanalyzer 2100 (Agilent

7 technologies, Santa Clara, CA, US). Qualified total RNA was further purified by

8 RNAClean XP Kit (Cat A63987, Beckman Coulter, Inc. Kraemer Boulevard

9 Brea, CA, USA) and RNase-Free DNase Set (Cat\#79254, QIAGEN, GmBH,

10 Germany).

\subsection{RNA Sequence and Data Analysis}

The preparation of the cDNA library from every ACC and spinal cord samples and the sequencing were performed by Shanghai Biotechnology Corporation and KangChen Biotechnology Corporation respectively. 6G raw data per sample were obtained on average. The unqualified sequencing reads were removed. And then Genome mapping were performed towards clean reads using HISAT2 tool ${ }^{27}$.In order to achieve gene expression standardization, clean reads were transformed into FPKM (Fragments Per Kilobase of exon model per Million mapped reads) ${ }^{28}$. Fold change was calculated according to

21 FPKM value. GO analysis and KEGG pathways of gene functional annotation clustering were performed by an $\mathrm{R}$ package which used a modified Fisher's 
1 exact test $^{29}$. Short Time-series Expression Miner analysis (STEM) were

2 applied to cluster time series gene expression data ${ }^{30}$. The PPI network

3 analysis was using STRING online software (http://string-db.org/). The ACC

4 and spinal cord sequencing results have been submitted to the Gene

5 Expression Omnibus (GEO) repository and assigned GEO accession number

6 as GSE172133 and GSE175760 respectively.

7

$8 \quad$ 5.6. Statistical Analysis

9 The GraphPad Prism 7 was used for statistical analysis. Data were analyzed

10 by two-way repeated measures ANOVA followed by Sidak's multiple

11 comparisons test. All data were presented as mean \pm SD and statistical

12 significance was set at $p<0.05$.

14 Ethics approval and consent to participate

$15 \quad$ Not applicable.

Consent for publication

Not applicable.

\section{Availability of data and materials}

21 All data generated or analyzed during this study can be found in online repositories. The names of the repositories and accession numbers can be 
1 found in the article.

3 Competing interests

4 The authors declare that they have no competing interests.

$6 \quad$ Funding

7 This work was supported by National Natural Science Foundation of China 8 (82071249 8177120781901264 and 81873733) and Hunan Province Clinical

9 Medical Technology Innovation Guide Project in 2018 (2018SK52602). The work of $Y Z$ was supported by the Fundamental Research Funds for the

11 Central Universities of Central South University.

\section{Author contributions}

$Y Z, Z H$ and $X Y$ carried out the experiments. SJ, LF, $X H$ and $Y Z$ analyzed data and presentation. $\mathrm{YZ}$ and $\mathrm{ZH}$ wrote the manuscript. QG participated in conception of the study. $\mathrm{CH}$ conducted the study design. All authors read and approved the final version of the manuscript.

\section{Acknowledgements}

Not applicable. 


\section{Reference}

1 1. Scholz J, Finnerup NB, Attal N, et al. The IASP classification of chronic pain for ICD-11: chronic neuropathic pain. Pain. 2019;160(1):53-59.

2. Taylor AM, Harris AD, Varnava $A$, et al. Neural responses to a modified Stroop paradigm in patients with complex chronic musculoskeletal pain compared to matched controls: an experimental functional magnetic resonance imaging study. $B M C$ psychology. 2016;4:5.

3. Buffington AL, Hanlon CA, McKeown MJ. Acute and persistent pain modulation of attention-related anterior cingulate fMRI activations. Pain. 2005;113(1-2):172-184.

4. Fomberstein K, Qadri S, Ramani R. Functional MRI and pain. Current opinion in anaesthesiology. 2013;26(5):588-593.

5. Zhuo M. Cortical excitation and chronic pain. Trends in neurosciences. 2008;31(4):199-207.

6. Luo C, Kuner T, Kuner R. Synaptic plasticity in pathological pain. Trends Neurosci. 2014;37(6):343-355.

7. Zhao MG, Ko SW, Wu LJ, et al. Enhanced presynaptic neurotransmitter release in the anterior cingulate cortex of mice with chronic pain. $J$ Neurosci. 2006;26(35):8923-8930.

8. $\mathrm{Xu} \mathrm{H}$, Wu LJ, Wang $\mathrm{H}$, et al. Presynaptic and postsynaptic amplifications of neuropathic pain in the anterior cingulate cortex. J Neurosci. 2008;28(29):7445-7453.

9. Ko HG, Choi JH, Park DI, et al. Rapid Turnover of Cortical NCAM1 Regulates Synaptic Reorganization after Peripheral Nerve Injury. Cell Rep. 2018;22(3):748-759.

10. $\mathrm{Li} \mathrm{XY}, \mathrm{Ko} \mathrm{HG}$, Chen T, et al. Alleviating neuropathic pain hypersensitivity by inhibiting PKMzeta in the anterior cingulate cortex. Science. 2010;330(6009):1400-1404.

11. Wang $\mathrm{H}, \mathrm{Xu} \mathrm{H}, \mathrm{Wu} \mathrm{LJ}$, et al. Identification of an adenylyl cyclase inhibitor for treating neuropathic and inflammatory pain. Science translational medicine. 2011;3(65):65ra63.

12. Bliss TV, Collingridge GL. Expression of NMDA receptor-dependent LTP in the hippocampus: bridging the divide. Molecular brain. 2013;6:5.

13. Bliss TV, Collingridge GL. A synaptic model of memory: long-term potentiation in the hippocampus. Nature. 1993;361(6407):31-39.

14. Bliss TV, Collingridge GL, Kaang BK, Zhuo M. Synaptic plasticity in the anterior cingulate cortex in acute and chronic pain. Nature reviews Neuroscience. 2016;17(8):485-496.

15. Koga K, Descalzi G, Chen T, et al. Coexistence of two forms of LTP in ACC provides a synaptic mechanism for the interactions between anxiety and chronic pain. Neuron. 2015;85(2):377-389.

16. Zhou Y, Lutz PE, Wang YC, Ragoussis J, Turecki G. Global long non-coding RNA expression in the rostral anterior cingulate cortex of depressed suicides. Translational psychiatry. 2018;8(1):224.

17. Akula N, Marenco S, Johnson K, et al. Deep transcriptome sequencing of subgenual anterior cingulate cortex reveals cross-diagnostic and diagnosis-specific RNA expression changes in major psychiatric disorders. Neuropsychopharmacology : 
official publication of the American College of Neuropsychopharmacology. 2021;46(7):1364-1372.

18. Kao CY, He Z, Zannas AS, et al. Fluoxetine treatment prevents the inflammatory response in a mouse model of posttraumatic stress disorder. Journal of psychiatric research. 2016;76:74-83.

19. Logan RW, Xue X, Ketchesin KD, et al. Sex Differences in Molecular Rhythms in the Human Cortex. Biological psychiatry. 2021.

20. Li M, Su S, Cai W, et al. Differentially Expressed Genes in the Brain of Aging Mice With Cognitive Alteration and Depression- and Anxiety-Like Behaviors. Frontiers in cell and developmental biology. 2020;8:814.

21. Su S, Li M, Wu D, et al. Gene Transcript Alterations in the Spinal Cord, Anterior Cingulate Cortex, and Amygdala in Mice Following Peripheral Nerve Injury. Frontiers in cell and developmental biology. 2021;9:634810.

22. Bennett GJ, Xie YK. A peripheral mononeuropathy in rat that produces disorders of pain sensation like those seen in man. Pain. 1988;33(1):87-107.

23. Shen Y, Ding Z, Ma S, et al. SETD7 mediates spinal microgliosis and neuropathic pain in a rat model of peripheral nerve injury. Brain Behav Immun. 2019;82:382-395.

24. Shen $\mathrm{Y}$, Ding Z, Ma S, et al. Targeting aurora kinase B alleviates spinal microgliosis and neuropathic pain in a rat model of peripheral nerve injury. $J$ Neurochem. 2020;152(1):72-91.

25. Wang D, An SC, Zhang X. Prevention of chronic stress-induced depression-like behavior by inducible nitric oxide inhibitor. Neurosci Lett. 2008;433(1):59-64.

26. Han M, Xiao X, Yang Y, et al. SIP30 is required for neuropathic pain-evoked aversion in rats. The Journal of neuroscience : the official journal of the Society for Neuroscience. 2014;34(2):346-355.

27. Pertea M, Kim D, Pertea GM, Leek JT, Salzberg SL. Transcript-level expression analysis of RNA-seq experiments with HISAT, StringTie and Ballgown. Nature protocols. 2016;11(9):1650-1667.

28. Mortazavi A, Williams BA, McCue K, Schaeffer L, Wold B. Mapping and quantifying mammalian transcriptomes by RNA-Seq. Nature methods. 2008;5(7):621-628.

29. Yu G, Wang LG, Han Y, He QY. clusterProfiler: an R package for comparing biological themes among gene clusters. Omics : a journal of integrative biology. 2012;16(5):284-287.

30. Ernst J, Bar-Joseph Z. STEM: a tool for the analysis of short time series gene expression data. BMC bioinformatics. 2006;7:191.

31. Wu XB, He LN, Jiang BC, Wang X, Lu Y, Gao YJ. Increased CXCL13 and CXCR5 in Anterior Cingulate Cortex Contributes to Neuropathic Pain-Related Conditioned Place Aversion. Neuroscience bulletin. 2019;35(4):613-623.

32. Harrison NA, Brydon L, Walker $C$, et al. Neural origins of human sickness in interoceptive responses to inflammation. Biological psychiatry. 2009;66(5):415-422.

33. Slavich GM, Way BM, Eisenberger NI, Taylor SE. Neural sensitivity to social rejection is associated with inflammatory responses to social stress. Proceedings of the National Academy of Sciences of the United States of America. 2010;107(33):14817-14822. 
34. Holmes SE, Hinz R, Conen S, et al. Elevated Translocator Protein in Anterior Cingulate in Major Depression and a Role for Inflammation in Suicidal Thinking: A Positron Emission Tomography Study. Biological psychiatry. 2018;83(1):61-69.

35. Miu J, Mitchell AJ, Müller $M$, et al. Chemokine gene expression during fatal murine cerebral malaria and protection due to CXCR3 deficiency. $J$ Immunol. 2008;180(2):1217-1230.

36. Kwiatkowski K, Piotrowska A, Rojewska E, et al. Beneficial properties of maraviroc on neuropathic pain development and opioid effectiveness in rats. Progress in neuro-psychopharmacology \& biological psychiatry. 2016;64:68-78.

37. Liou JT, Yuan HB, Mao CC, Lai YS, Day YJ. Absence of C-C motif chemokine ligand 5 in mice leads to decreased local macrophage recruitment and behavioral hypersensitivity in a murine neuropathic pain model. Pain. 2012;153(6):1283-1291.

38. Liou JT, Mao CC, Ching-Wah Sum D, et al. Peritoneal administration of Met-RANTES attenuates inflammatory and nociceptive responses in a murine neuropathic pain model. The journal of pain. 2013;14(1):24-35.

39. Wu XB, He LN, Jiang BC, et al. Spinal CXCL9 and CXCL11 are not involved in neuropathic pain despite an upregulation in the spinal cord following spinal nerve injury. Mol Pain. 2018;14:1744806918777401.

40. Raghu H, Lepus CM, Wang Q, et al. CCL2/CCR2, but not CCL5/CCR5, mediates monocyte recruitment, inflammation and cartilage destruction in osteoarthritis. Ann Rheum Dis. 2017;76(5):914-922.

41. Tacke F, Alvarez D, Kaplan TJ, et al. Monocyte subsets differentially employ CCR2, CCR5, and CX3CR1 to accumulate within atherosclerotic plaques. $J$ Clin Invest. 2007;117(1):185-194.

42. Pawlik K, Piotrowska A, Kwiatkowski K, et al. The blockade of CC chemokine receptor type 1 influences the level of nociceptive factors and enhances opioid analgesic potency in a rat model of neuropathic pain. Immunology. 2020;159(4):413-428.

43. Wennerberg K, Der CJ. Rho-family GTPases: it's not only Rac and Rho (and I like it). $J$ Cell Sci. 2004;117(Pt 8):1301-1312.

44. Yang YK, Lu XB, Wang YH, Yang MM, Jiang DM. Identification crucial genes in peripheral neuropathic pain induced by spared nerve injury. European review for medical and pharmacological sciences. 2014;18(15):2152-2159.

45. Wang J, Ma SH, Tao R, Xia LJ, Liu L, Jiang YH. Gene expression profile changes in rat dorsal horn after sciatic nerve injury. Neurological research. 2017;39(2):176-182.

46. Diebold BA, Bokoch GM. Molecular basis for Rac2 regulation of phagocyte NADPH oxidase. Nature immunology. 2001;2(3):211-215.

47. Dooley JL, Abdel-Latif D, St Laurent CD, Puttagunta L, Befus D, Lacy P. Regulation of inflammation by Rac2 in immune complex-mediated acute lung injury. American journal of physiology Lung cellular and molecular physiology. 2009;297(6):L1091-1102.

48. Zotova E, Holmes C, Johnston D, Neal JW, Nicoll JA, Boche D. Microglial alterations in human Alzheimer's disease following A 442 immunization. Neuropathology and applied neurobiology. 2011;37(5):513-524.

49. Haruwaka K, Ikegami A, Tachibana $Y$, et al. Dual microglia effects on blood brain 
50. Bui TM, Wiesolek HL, Sumagin R. ICAM-1: A master regulator of cellular responses in inflammation, injury resolution, and tumorigenesis. Journal of leukocyte biology. 2020;108(3):787-799.

51. Jean-Toussaint R, Tian Y, Chaudhuri AD, Haughey NJ, Sacan A, Ajit SK. Proteome characterization of small extracellular vesicles from spared nerve injury model of neuropathic pain. Journal of proteomics. 2020;211:103540.

52. Machelska $H$, Mousa SA, Brack A, et al. Opioid control of inflammatory pain regulated by intercellular adhesion molecule-1. J Neurosci. 2002;22(13):5588-5596.

53. Chen J, Zhang B, Pan C, Ren L, Chen Y. [Effects of monocyte chemotactic protein-3 on ICAM-1, VCAM-1, TF, and TFPI expression and apoptosis in human umbilical vein endothelial cells]. Nan Fang Yi Ke Da Xue Xue Bao. 2013;33(1):86-92.

54. Gu W, Yao L, Li L, et al. ICAM-1 regulates macrophage polarization by suppressing MCP-1 expression via miR-124 upregulation. Oncotarget. 2017;8(67):111882-111901.

55. Sousa C, Golebiewska A, Poovathingal SK, et al. Single-cell transcriptomics reveals distinct inflammation-induced microglia signatures. EMBO Rep. 2018;19(11).

56. Gris D, Marsh DR, Oatway MA, et al. Transient blockade of the CD11d/CD18 integrin reduces secondary damage after spinal cord injury, improving sensory, autonomic, and motor function. J Neurosci. 2004;24(16):4043-4051.

57. Tsuda M, Koga K, Chen T, Zhuo M. Neuronal and microglial mechanisms for neuropathic pain in the spinal dorsal horn and anterior cingulate cortex. Journal of neurochemistry. 2017;141(4):486-498.

58. Chen T, Koga K, Descalzi G, et al. Postsynaptic potentiation of corticospinal projecting neurons in the anterior cingulate cortex after nerve injury. Molecular pain. 2014;10:33.

59. Chen T, Wang W, Dong YL, et al. Postsynaptic insertion of AMPA receptor onto cortical pyramidal neurons in the anterior cingulate cortex after peripheral nerve injury. Molecular brain. 2014;7:76.

60. Basbaum Al, Fields HL. Endogenous pain control systems: brainstem spinal pathways and endorphin circuitry. Annual review of neuroscience. 1984;7:309-338.

61. Cohen SP, Mao J. Neuropathic pain: mechanisms and their clinical implications. BMJ. 2014;348:f7656.

62. Nie $\mathrm{Y}$, Huang $\mathrm{H}$, Guo $\mathrm{M}$, et al. Breast Phyllodes Tumors Recruit and Repolarize Tumor-Associated Macrophages via Secreting CCL5 to Promote Malignant Progression, Which Can Be Inhibited by CCR5 Inhibition Therapy. Clinical cancer research : an official journal of the American Association for Cancer Research. 2019;25(13):3873-3886.

63. Piotrowska A, Kwiatkowski K, Rojewska E, Makuch W, Mika J. Maraviroc reduces neuropathic pain through polarization of microglia and astroglia - Evidence from in vivo and in vitro studies. Neuropharmacology. 2016;108:207-219.

64. Hoggatt J, Singh P, Tate TA, et al. Rapid Mobilization Reveals a Highly Engraftable Hematopoietic Stem Cell. Cell. 2018;172(1-2):191-204.e110.

65. Liu ZY, Song ZW, Guo SW, et al. CXCL12/CXCR4 signaling contributes to neuropathic pain via central sensitization mechanisms in a rat spinal nerve ligation model. CNS 
neuroscience \& therapeutics. 2019;25(9):922-936.

2 66. Kasamon $\mathrm{YL}$, Chen $\mathrm{H}$, de Claro RA, et al. FDA Approval Summary: Mogamulizumab-kpkc for Mycosis Fungoides and Sézary Syndrome. Clinical cancer research : an official journal of the American Association for Cancer Research. 2019;25(24):7275-7280. 


\section{$1 \quad$ Figure legends}

2 Figure 1. Behavioral Characterization of The Rats After $\mathrm{CCl}$

3 Mechanical allodynia (A) and thermal hyperalgesia (B)were induced after $\mathrm{CCl}$

4 surgery $(n=8)$. C. Sucrose preference test $(n=8)$. D. $C C l$ rats spent more time

5 in the light area than sham rats in the PEAP test $(n=6)$. Results are

6 represented as means $\pm \mathrm{SD}$. Two-way ANOVA; ${ }^{*} \mathrm{P}<0.05$ vs sham group, ${ }^{* * *} \mathrm{P}$

$7<0.001$ vs sham group.

9 Figure 2. The quality control of raw RNA-seq data set about ACC

A. Heatmap of the correlation between each sample with the Pearson test. B.

The summary of quality control of raw RNA-seq data set. It shows Raw reads, Clean reads, Clean ratio, Mapping ratio and Q20 (Phred quality scores Q) of 18 samples.

Figure 3. Transcripts Regulated in the ACC of Rat After CCI

A-E. Volcano plots of all the DEGs from sham group compared to $\mathrm{CCl}$ groups. Log2(fold change) is plotted as the abscissa and $-\log 10(\mathrm{P}$ Value) is plotted as the ordinate. up-regulated genes are indicated in red and down-regulated genes are indicated in green. The gray dots represent genes with no significant difference. F. Venn diagram showing the number of unique and

21 shared DEGs meeting fold change $>1.5$ and $P$-value $<0.05$ in each time point.

G. Histogram showing the statistics of up- and down-regulated DEGs in each 
1 time point.

3 Figure 4. Gene Ontology Analysis of DEGs

4 GO analysis showed the top 30 significantly enrichments of DEGs in biological

$5 \quad$ process $(\mathbf{A})$, cellular component $(\mathbf{B})$ and molecular function $(\mathbf{C})$. The $\mathrm{GO}$ terms

6 were plotted as the ordinate and the gene number was plotted as the abscissa.

8 Figure 5. KEGG Analysis of DEGs

9 The comparison of pathway enrichment in the ACC of rats after $\mathrm{CCl}$. It showed the top 30 significantly enriched KEGG pathways. The KEGG terms were

11 plotted as the ordinate and the rich factor is plotted as the abscissa. The size 12 of the dots represented the gene number.

14 Figure 6. Short Time-series Expression Miner analysis of DEGs in ACC after $\mathrm{CCl}$

A. Model profiles of time series gene expression. The data was sampled at six time points $0 d($ sham), $12 h, 1 d, 3 d, 7 d$ and $14 d$. The profile ID number was in the top left-hand corner and the profiles with statistical differences were shown

19 in color.

B. Significant model profiles provided detailed information about a K-means cluster. 
$1 \quad$ Figure 7. PPI Network Analysis of DEGs

2 STRING analysis for PPI Network of DEGs in profile 27 and 36. Network

3 nodes represent proteins. Edges represent protein-protein associations

4 including known interactions, predicted interactions, textmining, co-expression

5 and protein homology. PPI enrichment $p$-value $<1.0 \mathrm{e}-16$.

7 Figure 8. Similar expressional changes of chemokines in ACC and spinal cord

8 A. Heatmap showing the expression patterns of genes. The down- and 9 up-regulated genes are presented as the indicated color bars (blue to red). B.

10 A bar chart showing the dynamic expression series of genes in ACC.

Table 1. Function analysis of DEGs in profile 27, 36 and 39. It shows the gene symbol of DEGs.

\section{Supplementary materiel}

Supplementary Figure1. Gene Ontology Analysis and KEGG Pathways of DEGs in profile 27,36 and 39

A. GO analysis showed the top 10 significantly enrichments of DEGs in biological process, molecular function and cellular component. The DEGs were from profile 27,36 and 39 respectively. The GO terms were plotted as the ordinate and the gene number was plotted as the abscissa. B. The comparison

22 of pathway enrichment in ACC of rats after $\mathrm{CCl}$. It showed the top 10 
1 significantly enriched KEGG pathways about DEGs in profile 27, 36and 39.

2 The KEGG terms were plotted as the ordinate and the rich factor is plotted as

3 the abscissa. The size of the dots represented the gene number.

4

5 Supplementary table. The summary of quality control of raw RNA-seq data set.

6 It shows Raw reads, Clean reads, Mapping ratio and Q30 of the spinal cord 7 samples. 


\section{Figure1}
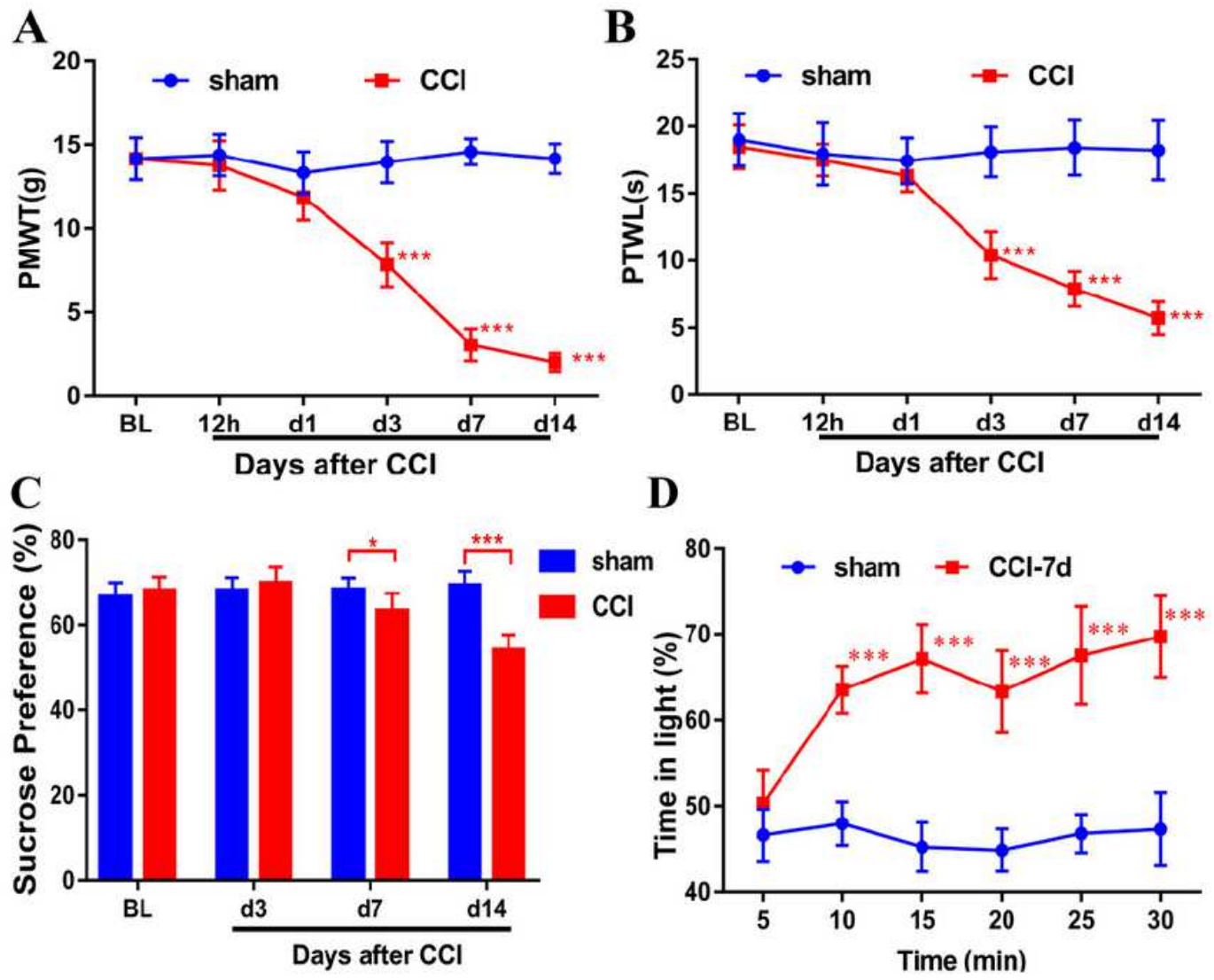

D Days after $\mathbf{C C l}$

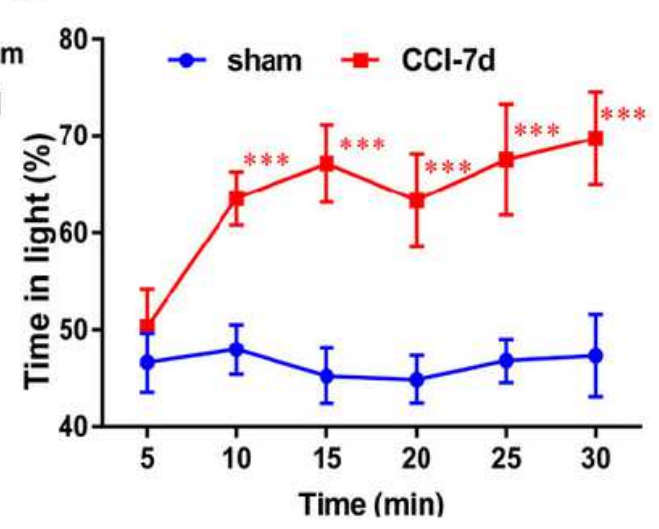

Figure 1

Behavioral Characterization of The Rats After CCI Mechanical allodynia (A) and thermal hyperalgesia (B)were induced after $\mathrm{CCl}$ surgery $(n=8)$. C. Sucrose preference test $(n=8) . D$. CCl rats spent more time in the light area than sham rats in the PEAP test $(n=6)$. Results are represented as means \pm SD. Two-way ANOVA; $* P<0.05$ vs sham group $\llbracket \star \star \star * P<0.001$ vs sham group. 


\section{Figure2}

A

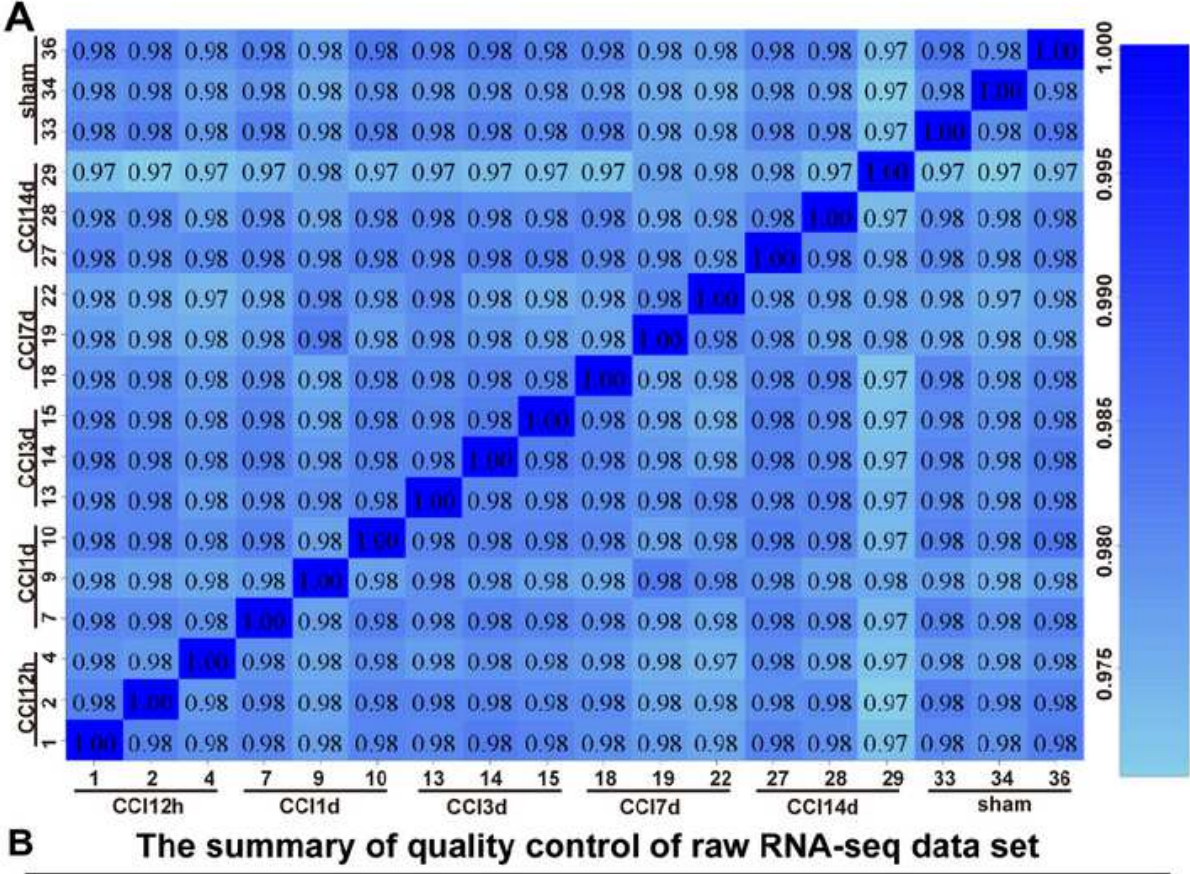

\begin{tabular}{|c|c|c|c|c|c|}
\hline Sample ID & Raw reads & Clean reads & Clean ratio & $\begin{array}{l}\text { Mapping } \\
\text { ratio }\end{array}$ & Q20 ratio \\
\hline 33-sham & $64,039,264$ & $61,494,620$ & $96.0 \%$ & $94.1 \%$ & $96.6 \%$ \\
\hline 34-sham & $39,705,288$ & $38,116,767$ & $96.0 \%$ & $94.1 \%$ & $96.4 \%$ \\
\hline 36-sham & $92,600,518$ & $87,322,307$ & $94.3 \%$ & $94.3 \%$ & $94.9 \%$ \\
\hline $1-\mathrm{CCl} 0.5 \mathrm{~d}$ & $40,268,996$ & $38,694,541$ & $96.1 \%$ & $94.3 \%$ & $96.6 \%$ \\
\hline 2-CCI0.5d & $56,908,130$ & $54,805,817$ & $96.3 \%$ & $94.0 \%$ & $96.5 \%$ \\
\hline 4-CCI0.5d & $54,974,980$ & $52,982,634$ & $96.4 \%$ & $94.2 \%$ & $96.5 \%$ \\
\hline 7-CCl1d & $52,391,704$ & $50,381,021$ & $96.2 \%$ & $94.4 \%$ & $96.4 \%$ \\
\hline 9-CCl1d & $56,291,374$ & $54,298,599$ & $96.5 \%$ & $94.0 \%$ & $96.7 \%$ \\
\hline $10-\mathrm{CCl} 1 \mathrm{~d}$ & $48,063,944$ & $45,576,056$ & $94.8 \%$ & $94.2 \%$ & $95.8 \%$ \\
\hline $13-\mathrm{CCl} 3 \mathrm{~d}$ & $65,371,216$ & $61,693,216$ & $94.4 \%$ & $94.0 \%$ & $96.6 \%$ \\
\hline $14-\mathrm{CCl} 3 \mathrm{~d}$ & $64,600,896$ & $62,228,090$ & $96.3 \%$ & $94.1 \%$ & $96.5 \%$ \\
\hline $15-\mathrm{CCl} 3 \mathrm{~d}$ & $53,445,186$ & $51,455,676$ & $96.3 \%$ & $94.2 \%$ & $95.0 \%$ \\
\hline $18-\mathrm{CCl} 7 \mathrm{~d}$ & $45,101,054$ & $43,315,065$ & $96.0 \%$ & $94.2 \%$ & $96.3 \%$ \\
\hline $19-\mathrm{CCl} 7 \mathrm{~d}$ & $50,898,078$ & $48,249,943$ & $94.8 \%$ & $94.2 \%$ & $95.3 \%$ \\
\hline 22-CCI7d & $88,276,058$ & $83,844,902$ & $95.0 \%$ & $93.9 \%$ & $95.8 \%$ \\
\hline 27-CCl14d & $77,588,642$ & $72,471,423$ & $93.4 \%$ & $94.2 \%$ & $94.4 \%$ \\
\hline 28-CCl14d & $64,512,466$ & $60,911,784$ & $94.4 \%$ & $94.1 \%$ & $95.0 \%$ \\
\hline 29-CCl14d & $61,014,052$ & $57,108,471$ & $93.6 \%$ & $94.1 \%$ & $94.7 \%$ \\
\hline
\end{tabular}

\section{Figure 2}

The quality control of raw RNA-seq data set about ACC A. Heatmap of the correlation between each sample with the Pearson test. B. The summary of quality control of raw RNA-seq data set. It shows Raw reads, Clean reads, Clean ratio, Mapping ratio and Q20 (Phred quality scores Q) of 18 samples. 


\section{Figure3}
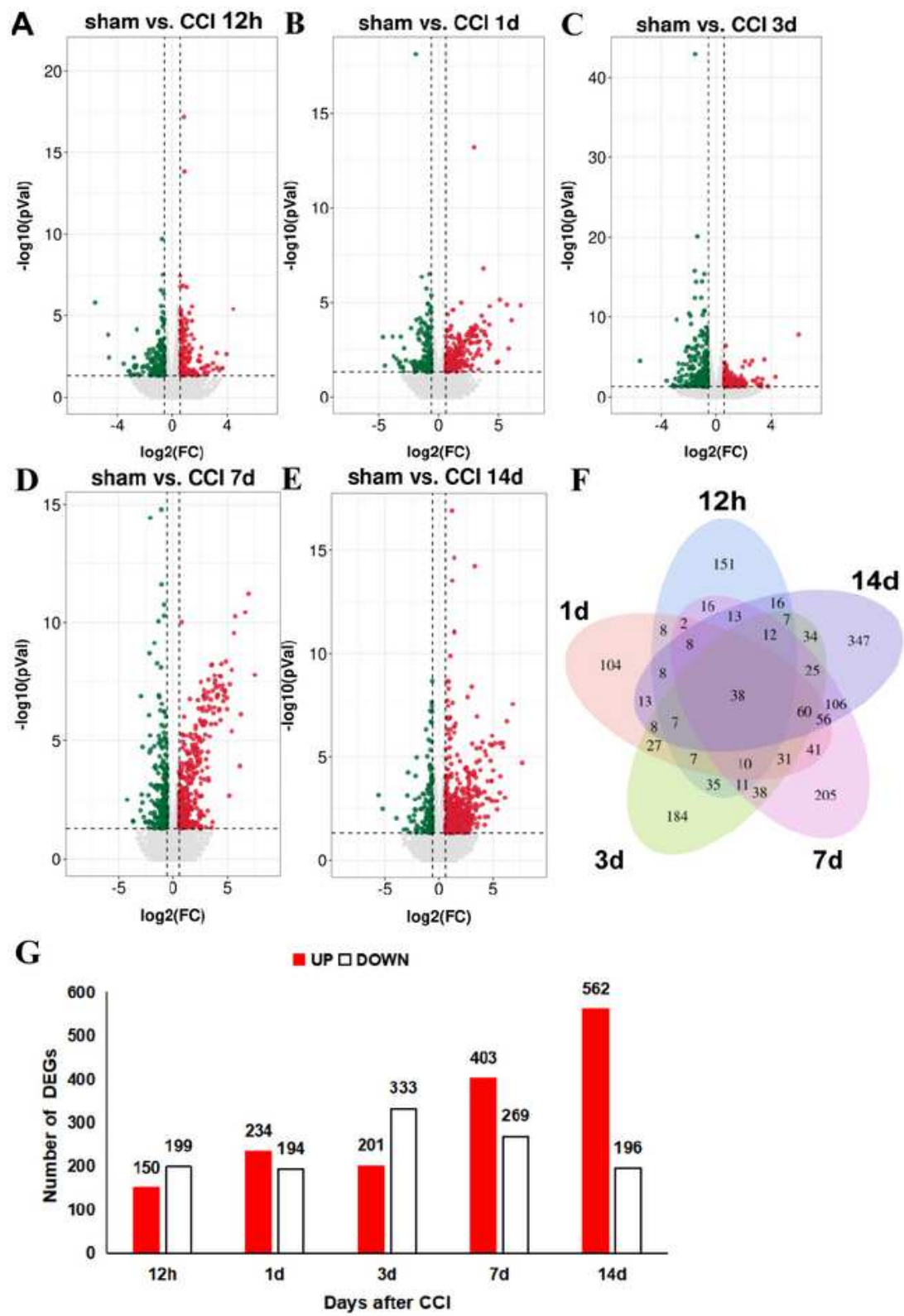

\section{Figure 3}

Transcripts Regulated in the ACC of Rat After CCI A-E. Volcano plots of all the DEGs from sham group compared to $\mathrm{CCl}$ groups. Log2(fold change) is plotted as the abscissa and -log10(P Value) is plotted as the ordinate. up-regulated genes are indicated in red and down-regulated genes are indicated in green. The gray dots represent genes with no significant difference. F. Venn diagram showing the number of 
unique and shared DEGs meeting fold change $>1.5$ and P-value $<0.05$ in each time point. G. Histogram showing the statistics of up- and down-regulated DEGs in each 1 time point

\section{Figure4}

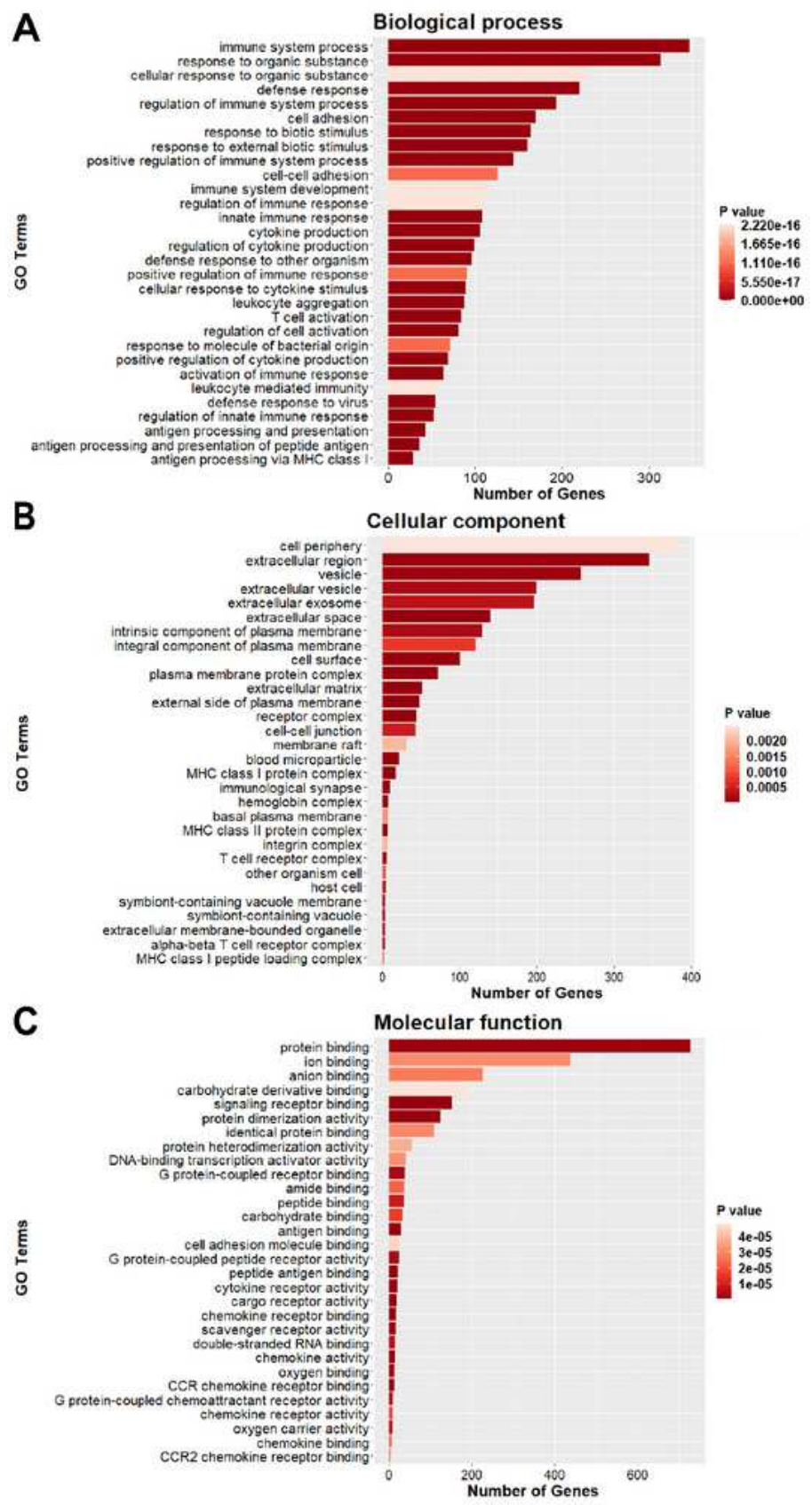

Figure 4

Gene Ontology Analysis of DEGs GO analysis showed the top 30 significantly enrichments of DEGs in biological process(A), cellular component (B) and molecular function (C). The GO terms were plotted as the ordinate and the gene number was plotted as the abscissa. 


\section{Figure5}

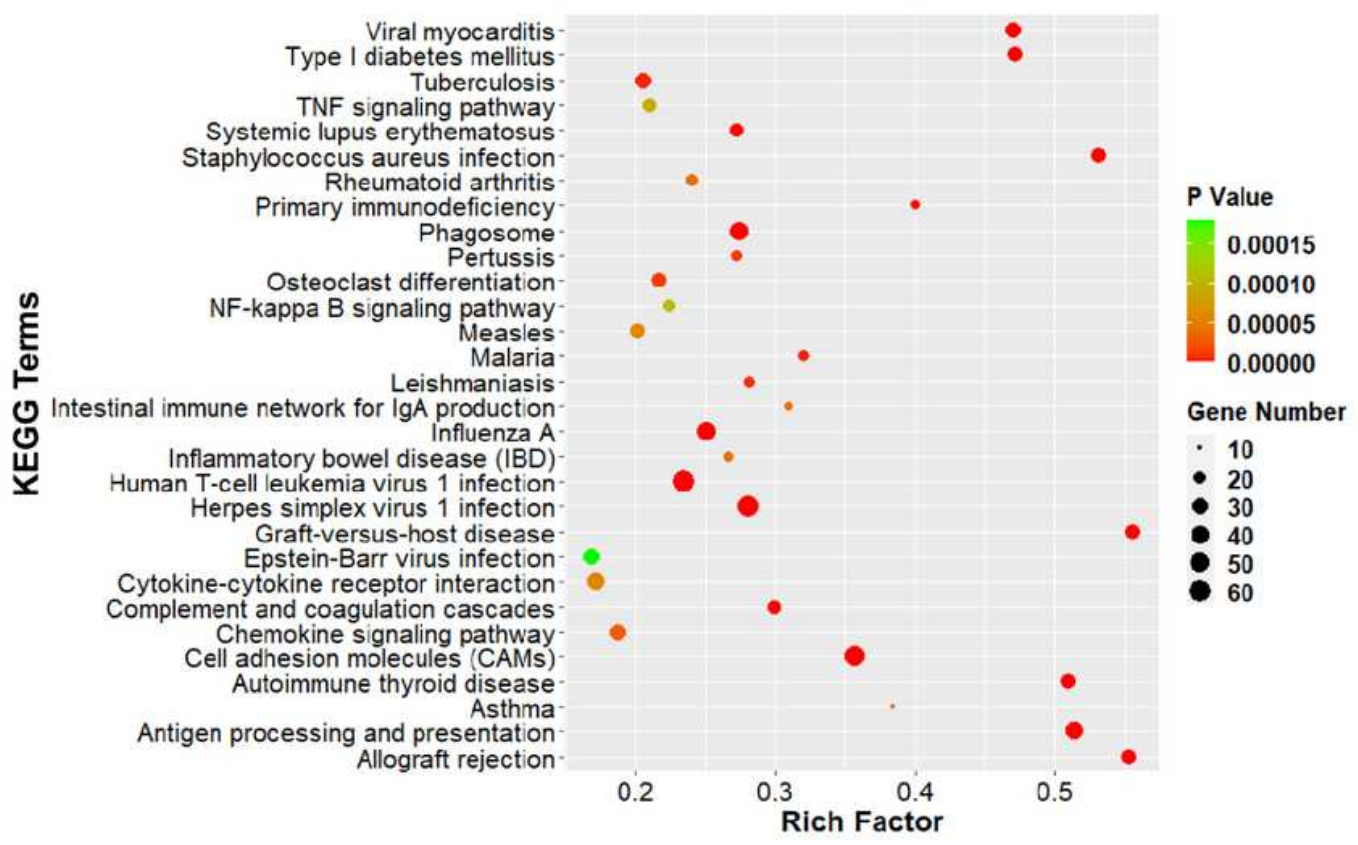

Figure 5

KEGG Analysis of DEGs The comparison of pathway enrichment in the ACC of rats after CCl. It showed the top 30 significantly enriched KEGG pathways. The KEGG terms were plotted as the ordinate and the rich factor is plotted as the abscissa. The size of the dots represented the gene number. 


\section{Figure6}

\section{A Profiles orderd by the p-value of significance of genes

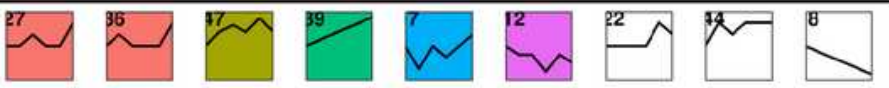
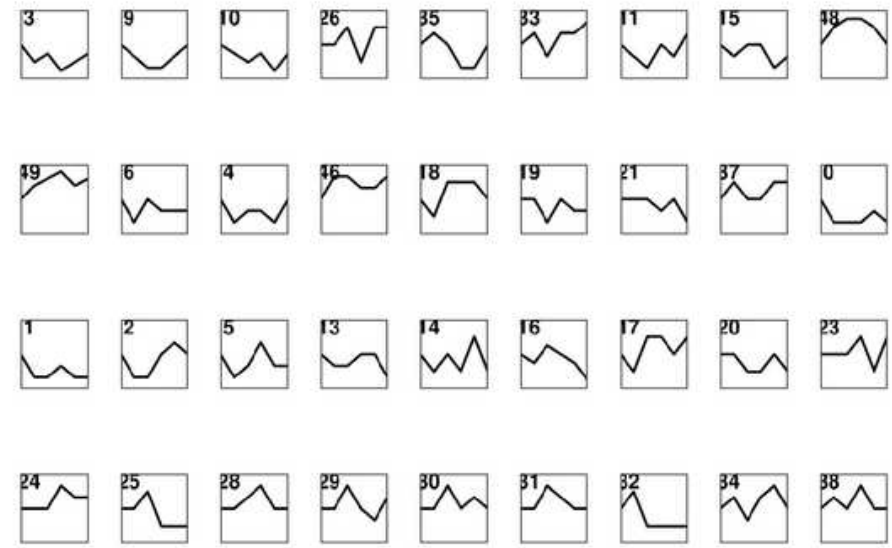
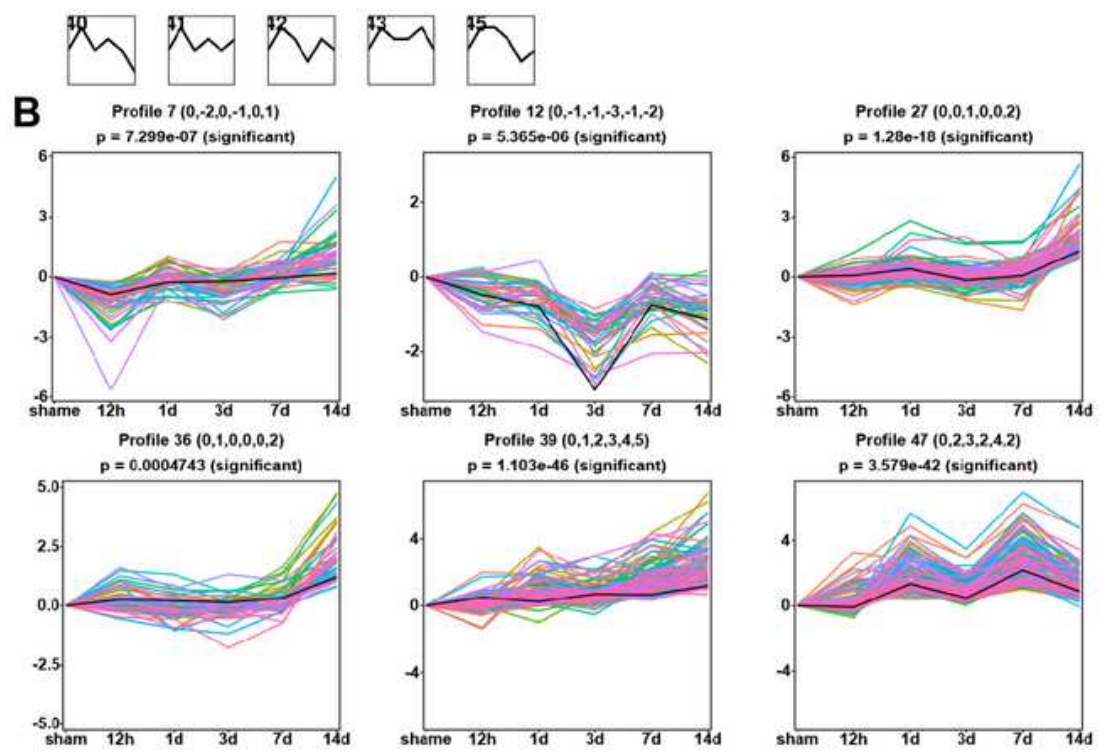

\section{Figure 6}

Short Time-series Expression Miner analysis of DEGs in ACC after CCI A. Model profiles of time series gene expression. The data was sampled at six time points $0 d($ sham), $12 \mathrm{~h}, 1 \mathrm{~d}, 3 \mathrm{~d}, 7 \mathrm{~d}$ and $14 \mathrm{~d}$. The profile ID number was in the top left-hand corner and the profiles with statistical differences were shown in color. B. Significant model profiles provided detailed information about a K-means cluster. 


\section{Figure7}

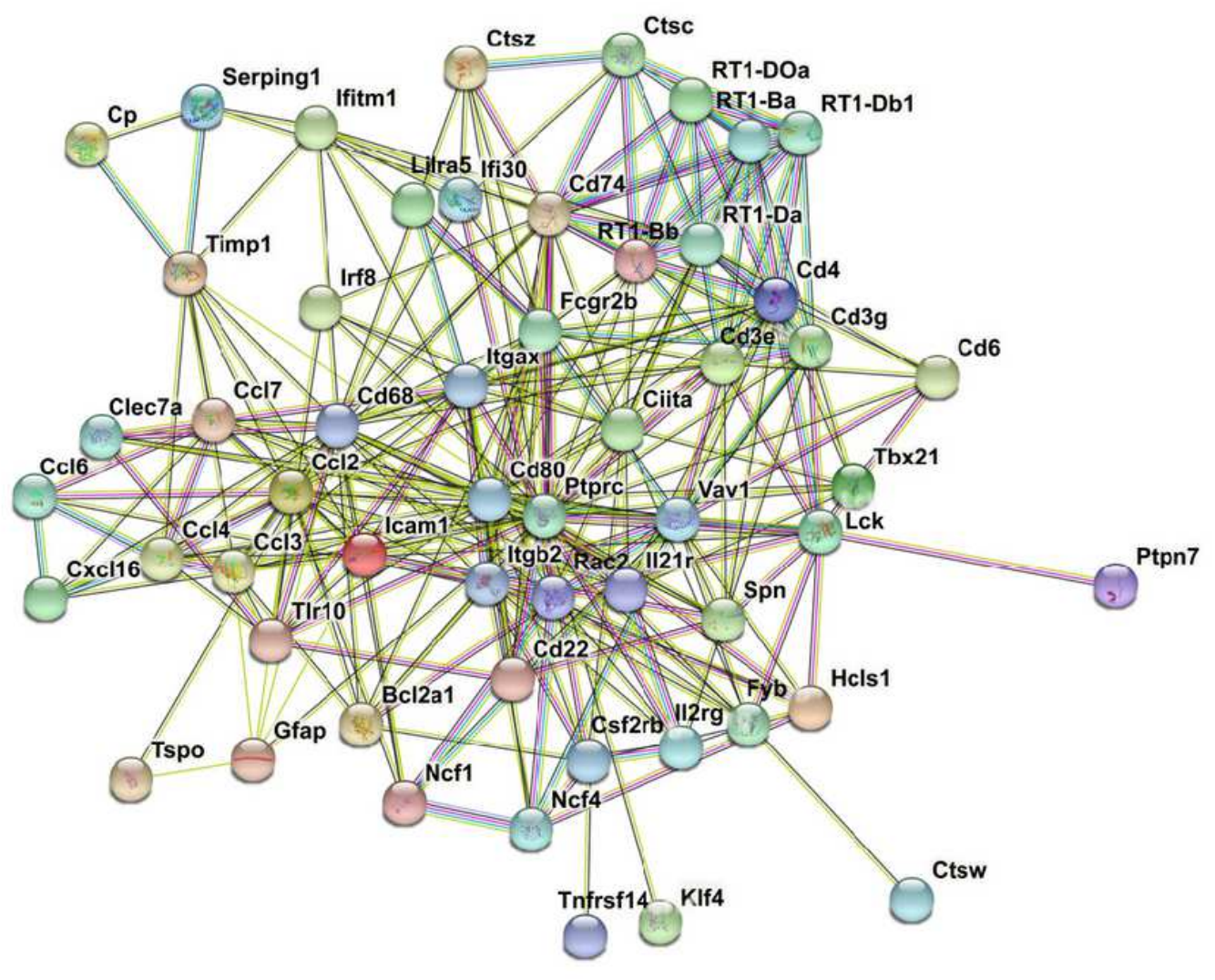

Figure 7

PPI Network Analysis of DEGs STRING analysis for PPI Network of DEGs in profile 27 and 36 . Network nodes represent proteins. Edges represent protein-protein associations including known interactions, predicted interactions, textmining, co-expression and protein homology. PPI enrichment $p$-value $<1.0 \mathrm{e}-16$. 
Figure8

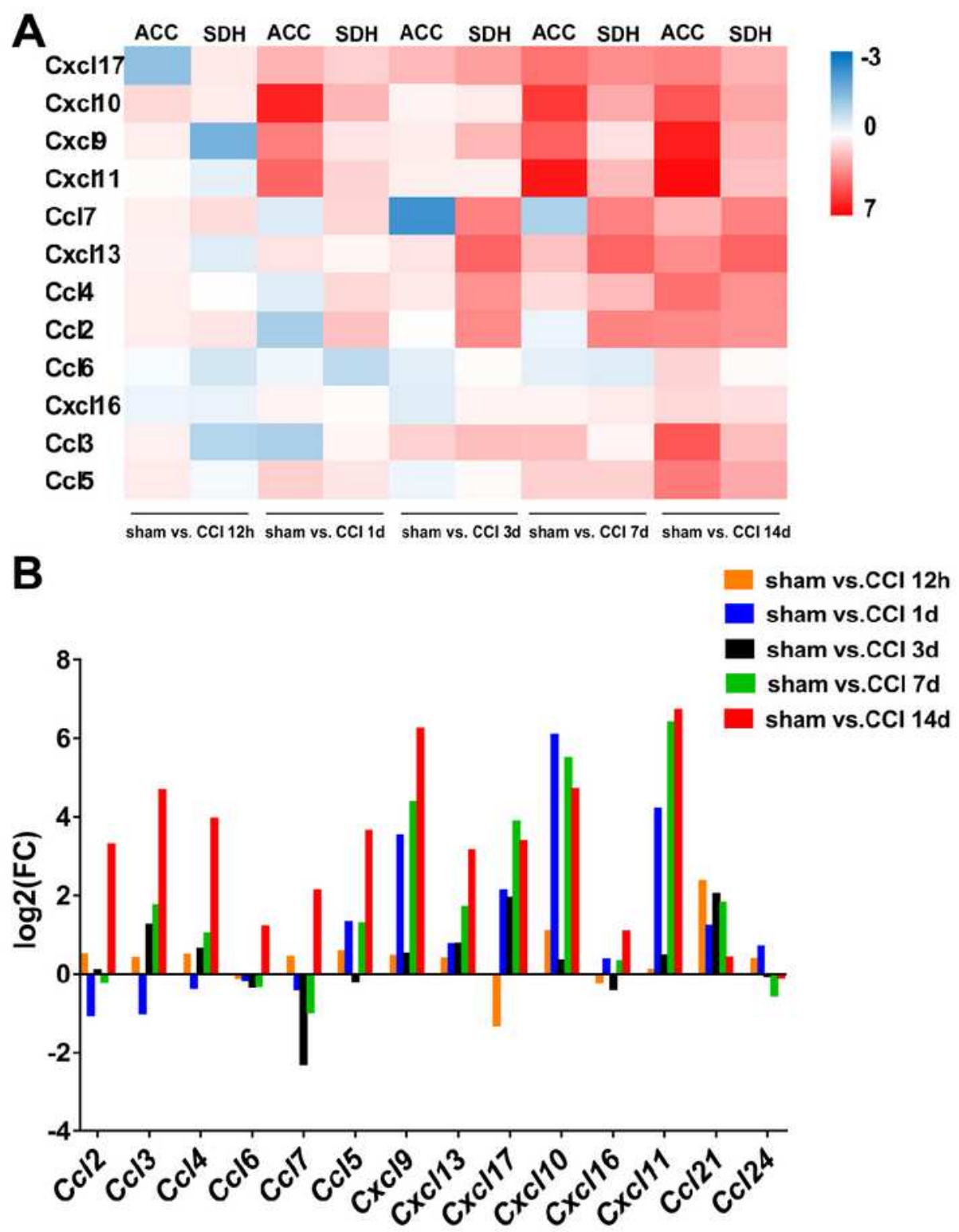

Figure 8

Similar expressional changes of chemokines in ACC and spinal cord A. Heatmap showing the expression patterns of genes. The down- and up-regulated genes are presented as the indicated color bars (blue to red). B. A bar chart showing the dynamic expression series of genes in ACC.

\section{Supplementary Files}


This is a list of supplementary files associated with this preprint. Click to download.

- SupplementaryMaterial.pdf

- Table.pdf 